AperTO - Archivio Istituzionale Open Access dell'Università di Torino

\title{
Obesity and peripheral neuropathy risk: a dangerous liaison
}

\section{This is the author's manuscript}

Original Citation:

Availability:

This version is available http://hdl.handle.net/2318/89831

since

Published version:

DOI:10.1111/j.1085-9489.2005.00047.x

Terms of use:

Open Access

Anyone can freely access the full text of works made available as "Open Access". Works made available under a Creative Commons license can be used according to the terms and conditions of said license. Use of all other works requires consent of the right holder (author or publisher) if not exempted from copyright protection by the applicable law. 


\title{
RESEARCHREPORT
}

\section{Obesity and peripheral neuropathy risk: a dangerous liaison}

\author{
Giacinta Miscio', Giulia Guastamacchia ${ }^{1,2}$, Amelia Brunani ${ }^{3}$, Lorenzo Priano', Silvia Baudo', \\ and Alessandro Mauro ${ }^{1,2}$ \\ ${ }^{1}$ Department of Neurology, Istituto Auxologico Italiano, IRCCS, Piancavallo (VB); ${ }^{2}$ Department of Neurosciences, University of \\ Turin, Turin; and ${ }^{3}$ Department of Metabolic Rehabilitation, Istituto Auxologico Italiano, IRCCS, Piancavallo (VB), Italy
}

\begin{abstract}
This study investigates motor (MNCS) and sensory (SNCS) nerve conduction in a sample of non-diabetic obese people without symptoms suggestive of neuropathy and looks for a possible metabolic alteration. Twenty-one patients and 20 age-matched controls underwent (a) MNCS (median, ulnar, peroneal, and tibial) and SNCS (median, ulnar, and sural); (b) quantitative sensory testing to measure sensory threshold for vibration, warm and cold sensation (WS-CS), heat and cold-induced pain; and (c) blood sample analysis to evaluate glucose and insulin levels and calculate the quantitative insulin-sensitivity check index (QUICKI). The obese group showed significantly decreased compound muscle action potential amplitude of tibial and peroneal nerves and decreased sensory action potential amplitude of all nerves. Most of the sensory thresholds were altered in obese patients. Insulin serum levels were significantly increased while QUICKI decreased in obese patients. WS and CS from the index and little fingers and WS from the big toe significantly correlated with QUICKI. Thermal and pain thresholds from the index and thermal thresholds from the little finger correlated with QUICKI values. The non-diabetic obese patients showed a subclinical involvement of different diameter sensory fibers. Such impairment was related to hyperinsulinemia and insulin sensitivity. The increase in sensory threshold of obese patients might be due to a metabolic alteration, potentially leading to a future clinical neuropathy.
\end{abstract}

Key words: diabetes, insulin resistance, neuropathy, obesity, sensory thresholds

\section{Introduction}

The thickness of the subcutaneous tissues in obese people has been held to account for the reduction in amplitude of sensory/mixed nerve responses when nerve conduction studies are performed using surface recording and percutaneous stimulation (Dumitru, 1995; Dorfman and Robinson, 1997; Buschbacher, 1998). Although this interpretation seems reasonable, obese people have also an increased risk for various metabolic

Address correspondence to: Giacinta Miscio, Department of Neurology and Neurorehabilitation, Istituto Auxologico Italiano, IRCCS, 'San Giuseppe' Hospital, Strada L. Cadorna 90, 28824 Piancavallo - Oggebbio (VB), Italy. Tel: +39-0323-514257x360; Fax: +39-0323-587694; E-mail: gmiscio@auxologico.it disorders, including impaired response to insulin in absence of increased blood glucose levels (Peters, 2000; Novella et al., 2001). In fact, insulin-sensitivity in non-diabetic persons is inversely correlated to body mass index (BMI) (Kahn et al., 1993), suggesting that obese patients develop increased insulin resistance. Nevertheless, to our knowledge, reports on nerve conduction studies in obesity lack any comments about these metabolic abnormalities.

In this prospective study, we had two aims to (1) investigate motor (MNCS) and sensory (SNCS) nerve conduction in a sample of non-diabetic obese patients without symptoms of neuropathy and (2) examine the relevance of the relationship between obesity and lowinsulin sensitivity for the development of a subclinical peripheral nerve damage. 


\section{Materials and Methods}

Twenty-one non-diabetic obese patients (mean age 38.95 years, range 20-60 years) with normal Achilles reflexes and sensation and without symptoms of peripheral nerve or root involvement and 20 age- and sex-matched controls (mean age 37.95 years, range 29-48 years) were recruited. Mean BMI was $41.06 \pm 4.74$ and $22.71 \pm 2.88$, respectively $(p \leq 0.0001)$. The mean height of the obese patients was $165.9 \pm 10.6 \mathrm{~cm}$ (range 150-188 cm) and that of the controls was $170.8 \pm 10 \mathrm{~cm}$ (range 155-182 cm) $(p=0.67)$. Exclusion criteria included other neurological or neuromuscular conditions, drugs, diabetes, thyroid disease, alcoholism, root lesions, symptoms of sensory dysfunction, and familial history of neuropathy. Diabetes was excluded according to American Diabetes Association criteria (American Diabetes Association, 2003), which defines a plasmatic basal glucose level higher than $126 \mathrm{mg} / \mathrm{dL}$ as a reliable indicator of diabetes. The study was approved by the local Ethical Committee.

A conventional neurographic study was performed to measure MNCS and/or SNCS in median, ulnar, peroneal, tibial, and sural nerves. SNCS was performed orthodromically for ulnar and median nerves, anti-dromically for the sural nerve, using disposable surface electrodes (Neuroline 700 10-SC). The distance between the stimulating electrode and $\mathrm{G} 1$ was kept constant in all the subjects. For MNCS, the onset latency, amplitude, and nerve conduction velocity were measured. For SNCS, the onset latency and peak to peak amplitude were measured. Skin surface temperature was measured over the dorsum of the hand and foot. The limb was warmed with a hairdryer if below $32^{\circ} \mathrm{C}$. Filter settings were $2 \mathrm{~Hz}$ to $10 \mathrm{kHz}$ and $20 \mathrm{~Hz}$ to $2 \mathrm{kHz}$, respectively, for motor and sensory recordings.

A computerized quantitative device (Medoc Ltd; TSA II-2001 and VSA-3000) was used to test the thresholds for vibratory sensation (Vib), warm and cold sensation (WS-CS), and heat- and cold-induced pain (HP-CP) (Dyck et al., 1993; Shy et al., 2003; Chong and Cros, 2004). The TSA-II-Neuro Sensory Analyzer and VSA-3000 Vibratory Sensory Analyzer are computerized non-invasive diagnostic tools for the quantitative assessment, respectively, of small caliber A-Delta/C and large caliber A-Beta sensory fiber dysfunction. A small thermal probe capable of heating or cooling was attached to the patient's skin. VSA-3000 measured the sensory threshold to vibration stimuli, between 0.1 and $130 \mu / \mathrm{s}$, by means of a vibratory platform on both hand and foot. According to the method of limits for both thermal and vibratory stimulation, the stimulus that progressively increases in intensity was halted by the subject with a simple push-button response as soon as sensation was perceived (Dyck et al., 1993; Shy et al., 2003). The analysis of HP and CP is not a pain-tolerance test. The patient is instructed to press the button when thermal sensation becomes unpleasant.

All subjects performed a sensitivity test composed of CS (range $32-0^{\circ} \mathrm{C}$ ), WS (range $32-50^{\circ} \mathrm{C}$ ), $\mathrm{CP}$ (range $32-0^{\circ} \mathrm{C}$ ), HP (range $32-50^{\circ} \mathrm{C}$ ), and vibratory sensation, six stimuli each and with a 4-s inter-stimulus interval to avoid adaptation. Thresholds were calculated on the mean value of each single test. Every test was performed on the plantar side of the big toe, hand palmar index, and little finger bilaterally.

Insulin resistance was determined in all subjects with the quantitative insulin-sensitivity check index (QUICKI) (Katz et al., 2000), calculated measuring the fasting insulin and glucose serum levels at baseline condition. This method was previously validated with the in vivo gold standard technique for insulin sensitivity, the hyperinsulinemic euglycemic clamp (Abbasi and Reaven, 2002).

\section{Statistical analysis}

Descriptive statistics (mean values \pm SD) were used. Mann-Whitney U-test was used to compare the neurophysiological data in obese people and control group; $p<0.05$ was the limit for a significant difference. The correlations between the neurophysiological data and BMI or QUICKI were tested by means of Pearson's regression analysis. The statistical program used was STATVIEW 5.0 (SAS Institute, Inc).

\section{Results}

Tables 1 and 2 summarize the findings for MNCS and SNCS study and for sensory thresholds, respectively, in obese patients and controls; the difference between the two groups is also reported ( $p$ value). Obese patients did not show a difference in glucose levels with respect to normal subjects but showed a significant increase in insulin serum levels and decreased QUICKI values as described in Table 3.

$\mathrm{BMI}$ was significantly correlated to QUICKI $(r=0.63, \quad p<0.0001)$. BMI highly correlated with motor and sensory latencies (M-ulnar nerve: $r=$ $-0.40, \quad p=0.0002 ; \quad M-t i b i a l$ nerve: $r=-0.36$, $\mathrm{p}=0.001$; M-peronal nerve: $r=-0.50, \mathrm{p}<0.0001$; SAP median nerve: $r=-0.31, p=0.008$; SAP ulnar nerve: $r=-0.43, p<0.0001$; and sural nerve: $r=$ $-0.33, p=0.002$ ) and namely to sensory response amplitudes (median nerve: $r=-0.59, p<0.0001$; ulnar nerve: $r=-0.45, p<0.0001$; and sural nerve: $r=-0.55, p<0.0001)$. No correlation was found 
Table 1. Comparison of the MNCS and SNCS in controls and obese patients (Mann-Whitney $U$-test).

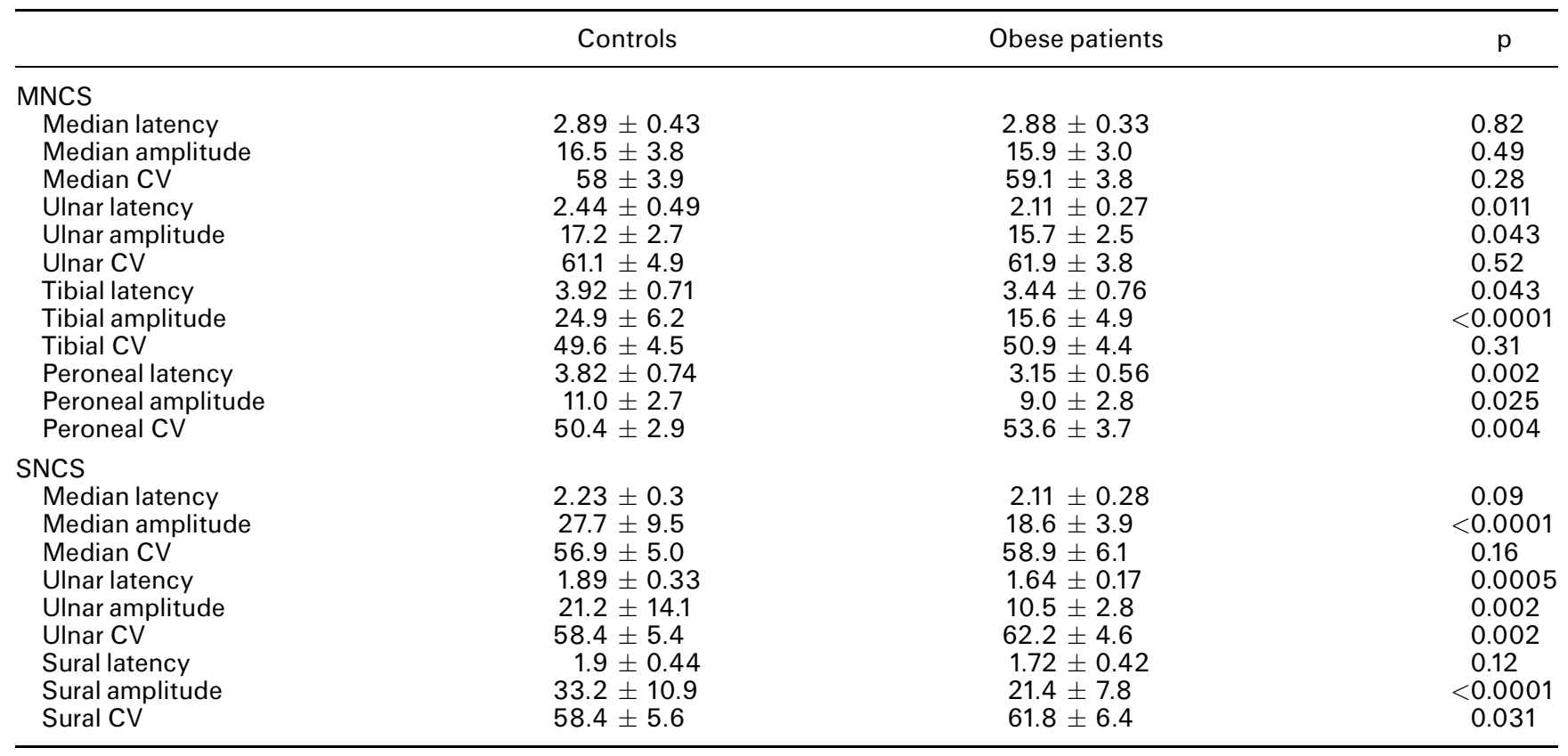

CV, conduction velocity; MNCS, motor nerve conduction study; SNCS, sensory nerve conduction study.

between compound muscle action potential-sensory nerve action potential parameters and QUICKI index.

Vibratory thresholds tended to be related to BMI but without reaching statistical significance (vibratory thresholds from index and little finger: $r=+0.40$, $p=0.02$ and $r=+0.37, p=0.02$, respectively). Any of the thermal and pain thresholds significantly correlated to BMI. Conversely, most of the thermal thresholds significantly correlated to QUICKI values (CS from index and little finger: $r=+0.50, p=0.004$ and $r=+0.42, p=0.009$, respectively; WS from index and little finger: $r=-0.43, p=0.01$ and $r=-0.46$, $p=0.003$, respectively; and WS from allux $r=-0.47$, $p=0.002)$. Pain threshold did not show correlation with QUICKI.

\section{Discussion}

This study confirms previous reports documenting the reduction of motor and sensory nerve-response amplitudes in obese people and its correlation to BMI (Dumitru, 1995; Dorfman and Robinson, 1997;

Table 2. Comparison of the sensory threshold findings in controls and obese patients (Mann-Whitney $U$-test).

\begin{tabular}{lccc}
\hline & Controls & Obese patients \\
\hline $\begin{array}{l}\text { Vibratory threshold } \\
\text { Index finger }\end{array}$ & & & \\
Little finger & $1.29 \pm 0.61$ & $2.66 \pm 4.25$ & 0.71 \\
Big toe & $1.32 \pm 0.68$ & $2.10 \pm 2.7$ & 0.87 \\
Thermal and pain threshold & $3.1 \pm 1.45$ & & \\
CS index finger & & & \\
WS index finger & $28.5 \pm 1.9$ & $28.6 \pm 3.1$ & 0.10 \\
CP index finger & $35.1 \pm 1.7$ & $36.6 \pm 3.8$ & 0.9 \\
HP index finger & $20.5 \pm 5.1$ & $46.4 \pm 3.6$ & 10.2 \\
CS little finger & $42.6 \pm 3.6$ & $27.5 \pm 3.7$ & 0.004 \\
WS little finger & $27.6 \pm 2.2$ & $37.7 \pm 3.3$ & 0.002 \\
CP little finger & $35.7 \pm 1.8$ & $13.1 \pm 10.1$ & 0.56 \\
HP little finger & $19.3 \pm 6.0$ & $46.2 \pm 3.9$ & 0.02 \\
CS big toe & $43.3 \pm 3.5$ & $26.1 \pm 3.0$ & 0.021 \\
WS big toe & $25.6 \pm 3.0$ & $41.5 \pm 3.7$ & 0.016 \\
CP big toe & $40.2 \pm 3.8$ & $43.5 \pm 9.8$ & 0.41 \\
HP big toe & $17.5 \pm 5.4$ & $48.1 \pm 3.0$ \\
\hline
\end{tabular}

$\mathrm{CP}$, cold pain; CS, cold sensation; HP, heat pain; WS, warm sensation. 
Table 3. Comparison of the metabolic findings in controls and obese patients (Mann-Whitney $U$-test).

\begin{tabular}{lccc}
\hline & Controls & Obese patients & \\
\hline Glucose levels $(\mathrm{mg} / \mathrm{dL})$ & $88.6 \pm 13.4$ & $86.6 \pm 8.2$ & $\mathrm{~N}$ \\
Insulin levels $(\mu \mathrm{U} / \mathrm{mL})$ & $5.7 \pm 2.0$ & $12.0 \pm 4.1$ & $<$ \\
QUICKI index & $0.378 \pm 0.032$ & $0.335 \pm 0.019$ & $<0.0001$ \\
\hline
\end{tabular}

NS, not significant; QUICKI, quantitative insulin-sensitivity check index.

Buschbacher, 1998). In fact, the thicker subcutaneous layer can dampen the amplitude of the nerve response when surface recording and percutaneous stimulation are used. The deeper location of the tibial nerve could account for the more consistent decrease in amplitude of this nerve in obese subjects (Buschbacher, 1998). We also found a significant reduction in some latency times of motor and sensory responses, according with the data by Buschbacher (1998). However, the relationship that we have confirmed between reduced motor/sensory amplitudes and BMI masks possible damage because of the metabolic derangement related to obesity.

Our study, in fact, showed a subclinical involvement also of different diameter sensory fibers in obese people: the HP thresholds from the index and little finger and the WS from the little finger were increased, while CP threshold from the upper limb districts was highly decreased. Such significant impairment of the thermal threshold was detected exclusively at the upper limb in contrast with the concept of a length-dependent affection in early neuropathy cases. It must be underlined that the quantitative computerized sensory threshold test was demonstrated to be more reliable if detected at the upper limb rather than at the lower limb (Bravenboer et al., 1992).

The alteration of the thermal thresholds was not related to $\mathrm{BMI}$, thus excluding an effect because of excessive weight. We then tried to find an alternative explanation, exploring the metabolic disorders linked to obesity. The key finding of the present study was the apparent exclusive relationship between thermal sensory threshold with hyperinsulinemia and reduced insulin sensitivity, data in favor of an initial involvement of the small nerve fibers. To our knowledge, the literature to date lacks any pathological evidence that obesity alone may cause neuropathy. Conversely, the relationship between obesity and impaired glycemic control is well known (Kahn et al., 1993). Recent studies indicate that abnormal increase in glucose is associated with a major risk of end-organ injury, including neuropathy (Feldman, 2003), and that impaired glucose tolerance is a causative factor in sensory neuropathy (Novella et al., 2001; Russell and Feldman, 2001; Singleton et al., 2001). The neuropathy associated with impaired glucose tolerance is milder than the neuropathy associated with diabetes.
Small nerve fibers are prominently affected and may be the earliest detectable sign of neuropathy in glucose dysmetabolism (Sumner et al., 2003).

In our sample of obese patients, blood glucose was in the normal range, but patients showed hyperinsulinemia and low-insulin sensitivity as documented by the decreased values of QUICKI. This parameter has been considered more sensitive than plasma insulin levels in predicting the onset of type 2 diabetes in obese subjects (Vanhala et al., 2002). The possible neurogenic impairment expressed by the altered sensory threshold was independent from glucose levels but related to hyperinsulinemia and insulin sensitivity. It follows that the increase in the sensory threshold demonstrated in some obese people might be due to a specific metabolic alteration, potentially leading to a future clinical neuropathy.

Our data also indicate that some nerve fibers might be more susceptible to damage than others, in particular, the small caliber or amyelinated fibers, while others with diameters large enough to sustain the normal conduction velocities may be spared. In this regard, we cannot exclude that the impaired insulin, as well as the altered glucose metabolism, may act on the $\mathrm{Na}^{+}$channels of the nodes of Ranvier of some fibers composing the nerve, disrupting their function (Brismar et al., 1987; Brismar, 1993).

In conclusion, our study, although conducted in a small sample of patients, documents subclinical peripheral nerve impairment in obesity. The possible interpretations of the derangement of some nerve fibers overcome the relationship already demonstrated between obesity and hyperglycemia or diabetes. In fact, the choice of the patients was very selective, to attribute a potential pathogenetic value to a metabolic alteration typical of the obese patients, represented by the reduced insulin sensitivity.

Further experimental studies based on a larger sample of obese patients, more accurate neurophysiological (i.e., near-nerve recording) and neuropathological (i.e., intraepidermal nerve fibers investigation) techniques, as well as animal models of obesity, would provide stronger support for the role of lowinsulin sensitivity as a risk factor for axonal impairment in obesity. 


\section{References}

Abbasi F, Reaven GM (2002). Evaluation of the quantitative insulin sensitivity check index as an estimate of insulin sensitivity in humans. Metabolism 51:235-237.

American Diabetes Association (2003). Screening for type 2 diabetes. Diabetes Care 26:S21-S24.

Bravenboer B, van Dam PS, Hop J, vd Steenhoven J, Erkelens DW (1992). Thermal threshold testing for the assessment of small fibre dysfunction: normal values and reproducibility. Diabet Med 9:546-549.

Brismar T (1993). Abnormal Na-currents in diabetic rat nerve nodal membrane. Diabet Med 10:110S-112S.

Brismar T, Sima AA, Greene DA (1987). Reversible and irreversible nodal dysfunction in diabetic neuropathy. Ann Neurol 21:504-507.

Buschbacher RM (1998). Body mass index effect on common nerve conduction study measurements. Muscle Nerve 21:1398-1404.

Chong PS, Cros DP (2004). Technology literature review: quantitative sensory testing. Muscle Nerve 29:734-747.

Dorfman LJ, Robinson LR (1997). AAEM minimonograph \#47: normative data in electrodiagnostic medicine. ff. Muscle Nerve 20:4-14

Dumitru D (1995). Nerve conduction studies. In: Electrodiagnostic Medicine. Dumitru D (Ed). Hanley and Belfus, Philadelphia, pp 111-209.

Dyck PJ, Karnes J, O'Brien PC, Zimmerman IR (1993). Detection thresholds of cutaneous sensation in humans. In: Peripheral Neuropathy. Dyck PJ (Ed). W.B. Saunders, Philadelphia, pp 706-728.

Feldman EL (2003). Oxidative stress and diabetic neuropathy: a new understanding of an old problem. J Clin Invest 111:431-433.

Kahn SE, Prigeon RL, McCulloch DK, Boyko EJ, Bergman RN, Schwartz MW, Neifing JL, Ward WK, Beard JC, Palmer JP,
Porte D Jr (1993). Quantification of the relationship between insulin sensitivity and beta-cell function in human subjects. Evidence for a hyperbolic function. Diabetes 42:1663-1672.

Katz A, Nambi SS, Mather K, Baron AD, Follmann DA, Sullivan G, Quon MJ (2000). Quantitative insulin sensitivity check index: a simple, accurate method for assessing insulin sensitivity in humans. J Clin Endocrinol Metab 85:2402-2410.

Novella SP, Inzucchi SE, Goldstein JM (2001). The frequency of undiagnosed diabetes and impaired glucose tolerance in patients with idiopathic sensory neuropathy. Muscle Nerve 24:1229-1231.

Peters AL (2000). The clinical implications of insulin resistance. Am J Manag Care 6:S668-S674.

Russell JW, Feldman EL (2001). Impaired glucose tolerance does it cause neuropathy? Muscle Nerve 24:1109-1112.

Shy ME, Frohman EM, So YT, Arezzo JC, Cornblath DR, Giuliani MJ, Kincaid JC, Ochoa JL, Parry GJ, Weimer LH; Therapeutics and Technology Assessment Subcommittee of the American Academy of Neurology (2003). Quantitative sensory testing: report of the Therapeutics and Technology Assessment Subcommittee of the American Academy of Neurology. Neurology 60:898-904.

Singleton JR, Smith AG, Bromberg MB (2001). Painful sensory polyneuropathy associated with impaired glucose tolerance. Muscle Nerve 24:1225-1228.

Sumner CJ, Sheth S, Griffin JW, Cornblath DR, Polydefkis M (2003). The spectrum of neuropathy in diabetes and impaired glucose tolerance. Neurology 60:108-111.

Vanhala P, Vanhala M, Kumpusalo E, Keinanen-Kiukaanniemi S (2002). The quantitative insulin sensitivity check index QUICKI predicts the onset of type 2 diabetes better than fasting plasma insulin in obese subjects; a 5-year follow-up study. J Clin Endocrinol Metab 87:5834-5837. 